\title{
Formación Profesional y gestión del saber. Los casos de la construcción y automotriz en Argentina
}

\author{
Vocational training and management knowledge. The case of construction and \\ automotive in Argentina
}

\author{
Pablo Granovsky \\ pgranovsky2004@yahoo.com.ar \\ Universidad de la Matanza, Argentina \\ Miguel Alfredo
malfredo@flacso.org.ar
Universidad de Buenos Aires, Argentina
}

Recepción: 23 Agosto 2019

Aprobación: 24 Agosto 2020

Publicación: 01 Septiembre 2020

Cita sugerida: Granovsky, P. y Alfredo, M (2020). Formación Profesional y gestión del saber. Los casos de la construcción y automotriz en Argentina. Archivos de Ciencias de la Educación, 14(17), e079. https://doi.org/10.24215/23468866e079

\begin{abstract}
Resumen: El siguiente artículo se propone aportar al debate de la política pública en Argentina a partir del papel que desarrolla el actor sindical dentro de la modalidad Formación Profesional. Retomando el desarrollo institucional de la misma, se describe el anclaje territorial y los perfiles profesionales desarrollados para dicha modalidad. En este punto, se busca problematizar sobre las particularidades que la construcción de las políticas de formación profesional asume en contextos de interacción con actores institucionales altamente especializados en los saberes y prácticas propias de las relaciones laborales y actividades sectoriales. Se recupera la experiencia de Formación Profesional gestionada por UOCRA (Unión Obrera de la Construcción de la República Argentina) y SMATA (Sindicato de Mecánicos y Afines del Transporte Automotor). A partir de entrevistas a informantes claves con inserción en la gestión de la Formación Profesional perteneciente al actor sindical y referentes técnicos, el artículo debate con interpretaciones ortodoxas y propone como marco analítico la acción pública y la sectorialización de la formación. El objetivo del artículo es aportar al debate entre el vínculo educación y trabajo a partir de la caracterización de la Formación Profesional como propio de gestión y dentro de un marco político-institucional situado.
\end{abstract}

Palabras clave: Política educativa, Formación Profesional, Gestión del saber, Sindicatos.

\begin{abstract}
The following article is proposed to contribute to the debate of public policy in Argentina based on the role played by the union actor in the Vocational Training modality. Taking up the institutional development of the same, the territorial anchorage and professional profiles developed for this modality are described. The experience of Professional Training managed by UOCRA (Construction Workers Union of the Republic of Argentina) and SMATA (Mechanics and Related Trade Union of Automotive Transport) is recovered. From interviews with key informants with insertion in the management of Vocational Training belonging to the union actor and technical referents, the article discusses with orthodox interpretations and proposes as an analytical framework the public action and the sectorialization of the training. The objective of the article is to contribute to the debate between the education and work link based on the characterization of Vocational Training as its own management and within a situated political-institutional framework.
\end{abstract}

Keywords: Educational policy, Vocational Training, Knowledge management, Unions. 


\section{INTRODUCCIÓN}

El artículo ${ }^{1}$ se propone aportar al debate de la política pública en Argentina a partir del papel que desarrolla el actor sindical dentro de la modalidad Formación Profesional. Analíticamente se debate con miradas simplistas propias de enfoques convencionales de la Administración de los RR.HH. La perspectiva desarrollada se distancia así de concepciones del desarrollo y la innovación ortodoxas que reducen los procesos de construcción de saber colectivo. Este enfoque simplista se aleja de las experiencias formativas y laborales donde, a partir de los procesos de aprendizaje, los agentes se transforman y se vuelven más diversos en relación con sus trayectorias, capacidades y saberes (Lundvall, 2009).

El concepto de acción pública permite dar cuenta del formato que asume la ejecución de una política pública a través de la mediación de los actores del mundo del trabajo. El funcionamiento de la red de Centros de Formación Profesional (CFP) y su entrecruzamiento con las políticas públicas convoca a pensar a la misma como un espacio de aprendizaje colectivo. En este sentido, se exploran las perspectivas sectoriales presentes en los referentes sindicales y las modalidades de ejecución de la política centrada en el sindicato como principal actor de las acciones. Se retoma la gestión institucional de la formación en el marco de los CFP de UOCRA (Unión Obrera de la Construcción de la República Argentina) y SMATA (Sindicato de Mecánicos y Afines del Transporte Automotor). Así, la arquitectura institucional, el entramado de actores y la red de compromisos, requieren de una visión y una práctica flexible en la articulación de estas políticas. En las entrevistas realizadas esta visión se orienta en el sentido de fortalecer la institucionalidad de las políticas para poder desarrollar un conjunto amplio de servicios y acciones de Formación Profesional. Esto supone el desarrollo de un saber experto en este campo y en la gestión de este tipo particular de instituciones y proyectos. Los testimonios analizados corresponden al trabajo de campo de las investigaciones de posgrado de los autores.

A partir del "recorte" sectorial de la política, la cercanía de las instituciones de formación con las prácticas laborales y tecnológicas propias del mundo del trabajo permite una orientación de la propuesta formativa con las figuras profesionales características de cada sector. En este punto, se busca problematizar sobre las particularidades que la construcción de las políticas de formación profesional asume en contextos de interacción con actores institucionales altamente especializados en los saberes y prácticas propias de las relaciones laborales y actividades sectoriales, siendo en el caso abordado, los informantes claves, gestores de la formación profesional propuesta por el actor sindical. De este modo, las preguntas que orientan el trabajo son: ¿Qué funciones desarrollan vinculadas a la gestión del Centro de Formación Profesional? ¿Qué aspectos observa de articulación-tensiones en relación con el vínculo entre el sistema educativo formal y los actores del mundo del trabajo? ¿Cuáles son los desafíos que afronta la Formación Profesional en torno a los avances tecnológicos?

En este sentido, se sostiene que la mirada sindical sectorial otorga un reconocimiento muy significativo a los saberes prácticos, lo que lleva a una instrumentación sectorial de las políticas de formación técnicoprofesional. Se caracterizan los procesos y prácticas formativas relevados con eje en la "gestión del saber" en el marco de estos dispositivos de formación, dando cuenta de lo que implica la ejecución de estas políticas en materia de planificación, ejecución y evaluación de estas. En cuanto a la construcción de compromisos institucionales para la formación, se desarrollan los aspectos vinculados a los elementos más amplios de estos 
dispositivos, en términos de la red de acuerdos institucionales a nivel "macro", así como del rol del actor sindical y sus modos de interpelación al actor estatal en el marco de una modalidad de acción pública.

\section{EDUCACIÓN Y TRABAJO. Dispositivos INSTITUCIONALES}

La cuestión de los vínculos entre la educación y el trabajo encuentra al interior de las ciencias sociales un vasto recorrido, dentro de las distintas vertientes de las corrientes críticas (reproductivismo, correspondencia), con sus matices y especificidades, existe una premisa fundamental: la producción del orden social es fruto de diversas trazas y espacios, entre los cuales la educación constituye un elemento clave a través del cual se conforman sujetos (Bourdieu y Passeron, 1977). La pugna por la distribución de bienes materiales y simbólicos lleva a que la consolidación de los programas pedagógicos y curriculares no se encuentre exenta de luchas, en el que la educación cumple diversos roles dentro de la lógica de reproducción del orden social (Filmus, 2014). Así, las contiendas entre perspectivas centradas en una educación generalizada y enciclopedista, frente a una formación específica y focalizada en un saber especializado y aplicable en labores productivas, es un marco histórico de interpretación básico para afrontar una política pública educativa (Gallart, 2002).

Considerando a la educación como un elemento constitutivo del sistema social pero además, y a su vez, como un campo de disputa, varios autores encuentran en la educación una visión histórico-social y la definen como producto de tensiones estructurales basadas en proyectos divergentes, los cuales se fundan en arraigadas tradiciones ideológico-culturales (Tedesco, 1986). La descolonización económica y cultural es un proceso pedagógico de aprehensión de la(s) realidad(es) (Wainsztok, 2013). En este sentido, la construcción de un sujeto pedagógico y del cuerpo escolar, junto a los contenidos, formas y normas curriculares, se constituyen en una lucha política por el control social del discurso escolar (Puiggrós y Carli, 1995), dentro del cual la legitimación de los saberes propios de las formaciones técnicas encuentra su propia especificidad (Giampaoletti, 2020). En este marco, la constitución de espacios de articulación y pugna refieren sustancialmente a relaciones sociales mediadas por diversos actores, entre los cuales, puntualmente en relación con la Formación Profesional en Argentina, es el actor sindical uno de los principales estandartes en quien se condensa la representación del sector trabajador aceptando/negociando/ imponiendo las condiciones a la hora de la definición del vínculo educación-trabajo (Baraldo, 2020).

De este modo, la educación confluirá en aspectos hegemónicos de carácter político, económico y/o político-económico, dependiendo de las exigencias y demandas sociales que ejerzan los diversos grupos sobre el Estado. A tal fin, la educación como proceso histórico se reconoce como un campo de disputa políticoideológico, donde sus posibilidades de factor democratizador dependerán del contexto socio-productivo y del ejercicio de las demandas políticas, en el cual las trayectorias educativo-laborales reflejan las tensiones propias de la reproducción/enclasamiento/transformación de la condición de origen (Miranda, 2016). A pesar de que numerosos trabajos e investigaciones han dado sobradas muestras de que las recetas únicas no son los márgenes más propicios para superar las condiciones de vulnerabilidad, la conceptualización de la educación como factor esencial para intervenir en el mejoramiento de las condiciones de vida, sobre todo en la de aquellos en donde las desregulaciones y la falta de presencia del Estado son más notorios, que es la población joven, resulta esencial (Tenti Fanfani, 2007; Dussel, 2020).

En esta línea, los argumentos sobre la necesidad de conformar y consolidar estrategias, herramientas y dispositivos institucionales para abonar a la formación y el empleo para sujetos en condiciones de vulnerabilidad resultan ampliamente abordados (Jacinto y Chitarroni, 2009; Millenaar, 2017). Particularmente, encauzado a jóvenes, Jacinto (2016) plantea que la orientación que tomaron los programas e iniciativas para el trabajo en América Latina vieron cambiar sus ejes rectores con respecto a la década de 1990, siendo que a partir de los 2000 las tendencias hacia la articulación entre educación formal, no formal 
y experiencias laborales han encontrado experiencias (como es el caso argentino) en donde la incorporación de nuevos actores otorgó mayores niveles de fortalecimiento de la institucionalidad (Briasco, 2014).

La instalación de sistemas nacionales y sectoriales de FP requiere ser pensada en su interacción con el campo científico tecnológico, con el mercado de trabajo, con el sistema educativo, con el mundo de las relaciones laborales y con cada sector productivo, siendo que su intervención puede ser conceptualizada como una acción pública (Verdier, 1997). En términos de Verdier (1997), el protagonismo de los actores del mundo del trabajo está dado en el desarrollo de equipos técnicos para instrumentar las acciones en donde el desarrollo de "acuerdos" resulta fundamental para la instalación de estos sistemas sectoriales. En el caso de la industria automotriz y de la industria de la construcción puede observarse como cierto marco estratégico-político con protagonismo sindical y con fuerte incidencia estatal en ambos casos, se da sustento a una propuesta formativa flexible y que responde a una demanda conformada por un entrecruzamiento de itinerarios diversos de los beneficiarios de las políticas de FP.

En este marco, resulta clave comprender el papel y el desenvolvimiento de los sistemas nacionales y sectoriales en su historia, tradiciones y desarrollo endógeno, ya que como sostiene Lundvall (2009), los sistemas de formación técnico profesional de carácter nacional juegan un papel clave al dar basamento político a los procesos de construcción de saber, en tanto instancia que permite la coordinación de las acciones, compartiendo un mismo ámbito comunitario -productivo, tecnológico y laboral-, reglas, normas y principios interpretativos comunes.

Caracterizar una red como la que se analiza en este artículo requiere enfocarse en la experiencia y la práctica de gestión de los proyectos de FP pensada como una "gestión sectorial del saber", sobre la que se estructura la política pública. Esta experiencia de gestión se relaciona con la formalización y socialización de saberes prácticos tanto en espacios formativos como productivos. De este modo, se entiende por gestión del saber aquellos procesos de diseño, planificación, instrumentación y monitoreo/seguimiento de las acciones tendientes a la construcción de saber experto y de capacidades tecnológicas y productivas de una comunidad así como de los mecanismos de su institucionalización, en donde el eje de esta está en el desarrollo de la formación y la calificación de la fuerza de trabajo. Esta gestión del saber implica una acción de política pública mediada por el protagonismo de los actores del mundo del trabajo (Neffa, 2000; Yoguel, 2000; Ferrer, 2004; Lundvall 2009).

En el caso argentino, y en un marco de fragmentación del sistema educativo formal, demandas del mercado de trabajo, de nuevas "capacidades y habilidades" y la superposición de las políticas sociales orientadas al empleo y a la capacitación laboral (Riquelme, 2006), orientarse al análisis de la relación entre el sistema educativo formal encadenado y la formación no encadenada, requiere pensar los diversos abordajes que desde los actores se llevan a cabo en pos de subsanar desigualdades existentes (Riquelme, Herger y Kodric, 2017). No obstante, el acceso diferenciado y los circuitos de calidad son elementos que a la hora de consolidar políticas de formación para el trabajo requieren tener los contextos socioeconómicos, ya que el lograr el reconocimiento de saberes productivos se consolida en los diversos itinerarios educativo-laborales (Corica y Otero, 2020).

\section{Aspectos Metodológicos}

El artículo busca a través de los testimonios de informantes claves poder reconstruir la lógica sectorial en la gestión del saber y la perspectiva gremial. Por tal motivo el entrecruzamiento entre "teoría" y "evidencia" se da a lo largo de todo el texto. El enfoque cualitativo resulta ser la herramienta de investigación implementada. Esta metodología y los campos de la educación y el trabajo cuentan con una vasta trayectoria (Miranda y Corica, 2018). Se adopta un enfoque cualitativo de investigación vinculándolo con la reconstrucción de las prácticas sociales de los actores de la FP, con el uso de métodos flexibles, inductivos, centrados en construir categorías conceptuales fundamentadas en la singularidad de los "casos" considerados. Se rescata el concepto de reflexión epistemológica, acercando la epistemología a la práctica de investigación, en el marco de la 
discusión sobre los paradigmas diversos presentes en las ciencias sociales (Vasilachis, 1992). La teoría resulta clave para la definición del encuadre de la investigación pensando en escenarios y relaciones macrosociales y/ o microsociales de la Formación P

El artículo busca a través de los testimonios de informantes claves poder reconstruir la lógica sectorial en la gestión del saber y la perspectiva gremial. Por tal motivo el entrecruzamiento entre "teoría" y "evidencia" se da a lo largo de todo el texto. El enfoque cualitativo resulta ser la herramienta de investigación implementada. Esta metodología y los campos de la educación y el trabajo cuentan con una vasta trayectoria (Miranda y Corica, 2018). Se adopta un enfoque cualitativo de investigación vinculándolo con la reconstrucción de las prácticas sociales de los actores de la FP, con el uso de métodos flexibles, inductivos, centrados en construir categorías conceptuales fundamentadas en la singularidad de los "casos" considerados. Se rescata el concepto de reflexión epistemológica, acercando la epistemología a la práctica de investigación, en el marco de la discusión sobre los paradigmas diversos presentes en las ciencias sociales (Vasilachis, 1992). La teoría resulta clave para la definición del encuadre de la investigación pensando en escenarios y relaciones macrosociales y/ o microsociales de la Formación Profesional, para la orientación de la investigación a partir del eje estructuraacción, y para formular esquemas explicativos centrados en la perspectiva del actor.

A partir de estas definiciones en el trabajo de campo se aplicaron distintas técnicas de aproximación a las prácticas de gestión y desarrollo de estas políticas sociales. Por un lado, se realizaron entrevistas en profundidad abiertas a partir de guías temáticas dirigidas a recuperar material espontáneo resultado de la interacción con el entrevistado en donde aquel pueda incluir temas, percepciones y valoraciones propias. Por otro, las entrevistas que se realizaron a referentes técnicos e institucionales de dichas instituciones. Las entrevistas fueron realizadas de forma individual en el ámbito del Área Metropolitana de Buenos Aires (AMBA). Así, se realizaron un total de 12 entrevistas que fueron llevadas a cabo durante el segundo semestre de 2017 y primer semestre de 2018.

\section{LA ACCIÓN PÚBLICA DE FORMACIÓN COMO ACCIÓN COMPLEJA}

Dar cuenta de las acciones de una red de CFP está relacionada con formas de articulación de normas, pautas y reglas con actores e instituciones para potenciar innovación productiva con integración social articulando una modalidad de estructuración de las relaciones laborales, formativas e industriales, en compromisos institucionalizados a nivel de los macro-actores: Estado, Trabajo y Mercado. A su vez, resulta pertinente analizar las formas de estructuración interna de la acción pública a nivel micro-organizacional y a nivel intermedio (red de instituciones y organizaciones).

La complejidad de esta gestión y del manejo institucional, abarca desde cuestiones de infraestructura e instalaciones, hasta lo pedagógico, desde la gestión de pedidos hasta la ampliación de la planta docente, entre otros. Las dificultades de la gestión afectan temáticas como el desarrollo, por parte de los equipos pedagógicos, de los materiales curriculares, así como la obtención de materiales e insumos.

La construcción de la política pública requiere de la participación de los actores en su instrumentación y ejecución. Para los casos abordados, implica encontrar las formas de desplegar las acciones que se correspondan con una segmentación y fragmentación de los trabajadores que asisten a los cursos. En otros términos, una "sintonía fina" en la articulación institucional y política de la acción pública, destacándose una dimensión pragmático-comunicativa que incluye como "normal” los conflictos y tensiones entre actores e instituciones. La complejidad que requiere esta integración de elementos diversos, de articulación compleja de distintos componentes, no puede basarse en principios de unicidad, lineales y "verticalistas", sino en el poder arribar a "pactos" parciales para la coordinación de las acciones entre actores heterogéneos, determinando objetivos y valores colectivos en un marco de diferencias y antagonismos, la búsqueda pragmática de acuerdos y compromisos parciales en torno a valores y perspectivas comunes, entre los agentes participantes. Este tipo de articulación dentro de las políticas de formación profesional y el tipo de 
racionalidad que planteamos a la política pública deben considerarse como intentos parciales por encontrar caminos alternativos al deterioro global y crisis de los acuerdos y compromisos asociados a la sociedad salarial y al periodo industrial. Desde esta mirada "macro", la base de la regulación del conflicto y de los dispositivos de integración social desarrollados en la FP, es un marco a partir del cual se instalaron un conjunto de protecciones dirigidas a los sectores subalternos -alumnos/trabajadores-, como un esquema de compensaciones que recaen sobre los sectores más desfavorablemente posicionados en la estructura social. Un intento de articulación de programas es el siguiente:

"En el sistema de formación profesional, en el sistema de jóvenes adultos, es el mismo público. ¿Por qué no hacemos un esfuerzo para ver si hay algunos contenidos que sean reconocidos en uno o en otro lado y que sean comunes? Que sean reconocidos para no hacerlos cursar dos veces. Entonces o que un contenido sea referido con el otro. Entonces, para dar un ejemplo, muy duro quizás, pero, cuando en un curso de electricidad están viendo un tablero eléctrico domiciliario vean superficie, la superficie del tablero, la maestra de primaria de geometría de superficie, lado por lado en secundaria, cuando veo en física, sea física aplicada a la electricidad, se refuerce eso y no tanto la velocidad de los cuerpos, no, no tanto velocidad sino electricidad. Entonces lo que estoy diciendo es: bueno, esto es una lógica cartesiana digamos, hacer que la persona tenga un reconocimiento; bueno hubo varias experiencias, hubo en Córdoba, en Mendoza, en la Pampa y en la fundación UOCRA. Hicieron estas experiencias justamente una de las experiencias, qué hizo UOCRA fue en el centro que ahora estoy dirigiendo..." (Javier, Director CFP UOCRA).

El despliegue territorial de las políticas de FP requiere integrar lo "productivo" con la integración social. Como ejemplo de búsquedas de acuerdo e integración con las provincias, uno de los entrevistados señalaba que en los dispositivos de la Educación Técnico Profesional existen coincidencias en el perfil de sus asistentes. Esto convoca a un esfuerzo por poder articular, en términos de sus contenidos formativos, con formas de reconocimiento que potencien el entrecruzamiento entre mundo laboral y los ingresos y egresos del trabajador al sistema formativo. Un esquema de oferta formativa flexible que se relacione de modo más fluido con la diversidad de trayectorias laborales existentes. Es en este sentido que se presentan las posibilidades de acercamiento entre los campos de la educación y el trabajo.

\section{LA SECTORIALIZACIÓN DE LA POLÍTICA PÚBLICA}

Esta gestión de los proyectos de FP implica en los referentes del sindicato asumir una perspectiva integral del sector que permita la instrumentación de mecanismos institucionales que potencien la "circulación" de saberes en el plano sectorial, organizacional e individual. En este sentido, se relaciona la formación con los procesos de gestión y control de calidad, cuidado medioambiental, actualización tecnológica y el desarrollo de oficios.

"Es el único sistema (FP) que cuenta con centros certificados en calidad educativa y que no existe en ninguna parte del sistema educativo y que tiene un régimen propio porque nosotros hicimos la normativa de formación profesional con el gobierno de la ciudad, con los sindicatos docentes, centrospropios del centro de la ciudad y centros sindicales y se hizo la reglamentación completa de lo que es la formación profesional en la ciudad de Buenos Aires. Abi te dicen todo: desde cómo nombras un profesional a la función que tiene cada uno, al tipo de certificados. Te dicen todo cómo tiene que ser y cómo tiene que trabajar la formación profesional. Eso es algo que también es destacableporque no todos los sistemas lo tienen, porque buscamos lo que es la transparencia" (Juan, director CFP SMATA).

A su vez se vincula con la promoción de la formación de saberes transferibles dentro de familias ocupacionales amplias. La arquitectura en que se expresa esta política pública y la "gestión sectorial del saber", se traducen en un conjunto de compromisos que suponen un estado de negociación permanente, lo que requiere el reconocimiento del lugar de los distintos interlocutores (Estado, sindicatos y empresas principalmente) como actores.

Los CFP que conforman una red pertenecen al sistema educativo público y las inversiones realizadas para su desarrollo son aprovechadas por toda la comunidad a la que pertenecen. Son instituciones de referencia tanto del sistema educativo como del sector productivo, ya que su operatividad se vincula con las 
necesidades emergentes de las transformaciones en la industria. Las acciones educativas que cada institución lleva adelante se realizan, según los entrevistados, desde una visión estratégica y un conocimiento profundo de las condiciones socio-productivas de las empresas del sector en la cual están inmersas. Estas políticas suponen el fortalecimiento institucional de un conjunto ampliado de acciones y servicios a partir de una compleja articulación de este dispositivo de política pública.

En cuanto al vínculo e inserción de las instituciones de FP con su comunidad, partiendo de estar "conveniadas" con el sindicato del sector, establecen relaciones con actores territoriales, por ejemplo, el municipio, promoviendo la formalización e institucionalización de sus acciones. Cabe resaltar que esta situación, si bien corresponde al momento actual y que en el pasado el trabajo conjunto entre ambas instituciones fue fluido, no es algo que sucede entre las organizaciones sindicales. En este marco, el carácter sectorial de las políticas y el papel de los actores del mundo del trabajo -la mediación de los actores en la ejecución de la política pública- aparece como uno de los elementos distintivos del dispositivo. Esto se manifiesta en la fuerte tensión contemporánea por reindividualizar las relaciones laborales o, por el contrario, buscar nuevas formas de "reinstitucionalizarlas", donde nuevos modos de regulación social permitan cierta "domesticación" de las fuerzas centrífugas del mercado, en una doble centralidad "moderna" del trabajo y el mercado. El caso de la Fundación UOCRA se orienta con claridad, en este segundo camino por la reinstitucionalización de las relaciones laborales a partir de su enfoque en la formación profesional como un aporte más a este proceso.

Una de las entrevistadas señalaba que el carácter sectorial de las políticas de FP se vincula con el papel de los actores del mundo del trabajo en las definiciones de los contenidos y las necesidades del sector de actividad respectivo, centralmente el actor sindical y el empresario, no tanto por los equipos técnicos del Ministerio de Trabajo. Manifiesta el lugar estratégico que desempeñan en el dispositivo los actores del mundo del trabajo. Lo específico de la función de los equipos técnicos es el de asistir en la instalación de los dispositivos pensando siempre en términos de "sistemas nacionales". De este modo, los aspectos metodológicos de indagación de estas demandas, a partir de un enfoque teórico-metodológico que articule intereses y perspectivas de los trabajadores y los empresarios, requiere la (re)consideración de la dinámica de los procesos productivos.

La lógica de la sectorialización de la política nacional de FP parte de un diagnóstico estatal, convocando luego a los actores privados y laborales. Así, según los entrevistados, la red de CFP se transforma en un sistema decisorio y de ejecución de la política pública, con un despliegue territorial y sectorial que responde a las demandas del mundo del trabajo y funciona como un dispositivo institucional de inserción. El eje en lo institucional es clave como "dispositivo de inserción" y su incidencia sobre los diversos modos de subjetivación en dicho marco. En un plano más general, estos entramados institucionales, a lo largo de la modernidad se configuran como soportes de modos y formatos de organizar la subjetividad obrera, sus itinerarios y sus esquemas referenciales de acción. Esto en un contexto global de "aflojamiento", debilitamiento y crisis de los vínculos sociales generados en los grandes marcos institucionales de integración y protección social (Jacinto, 2010). Dos enfoques entran en tensión a partir de este marco. El primero, centrado en un enfoque promercado, lo asocia a un cambio de época, el segundo lo aborda como una posibilidad de encontrar nuevas formas de reinstitucionalización de las relaciones laborales y del mundo del trabajo en contextos postfordistas y post sociedad salarial. En el primer caso, la activación responde a cierta descolectivización de las relaciones laborales y del mundo del trabajo, responsabilizando al sujeto beneficiario de su propio progreso personal. Por el contrario, desde la otra mirada, la activación supone esquemas más complejos de política pública, no acotados al mero subsidio, sino incentivando la participación; que los sujetos participen en un desarrollo activo de su vinculación o revinculación con el mundo del trabajo y que este vínculo sea concebido como un derecho.

Relacionando las políticas de FP en el marco más amplio de las políticas activas de empleo, uno de los entrevistados señalaba como fueron complementando las acciones con cierto cambio en la "territorialización" y federalización de la política, siendo que tradicionalmente el enfoque se encontraba 
centrado en Buenos Aires. Lo interesante es que la sectorialización se complementa y profundiza con esta territorialización, porque los actores sectoriales, principalmente el sindicato, a través de sus seccionales y delegaciones, es el que demanda la expansión territorial y el desarrollo de dispositivos formativos en todo el país. Dicho de otro modo, se flexibiliza la propuesta de política pública en función de la diversidad y heterogeneidad de demandas territoriales y sectoriales.

\section{GeSTión GREMIAL DEL SABER}

La gestión del saber conlleva tanto aspectos externos (como son los procesos de aprendizaje y la articulación con esquemas institucionales plurales) como una perspectiva pedagógica particular (apreciación de saberes preexistentes, ejercicio del aprendizaje en contextos situados, etc.). La capacidad de los actores involucrados en la gestión de sus redes de CFP encuentra en la canalización y validación institucional de antagonismos y conflictos un mecanismo para minimizar procesos de segregación interna. Un ejemplo de este enfoque, para el caso de la red de la UOCRA, donde puede verse la complejidad del manejo de las tensiones al interior de la red de formación, tiene que ver con la comunicación y difusión de las acciones en la red. El entrevistado explayó como participan tanto la institución de FP propiamente dicha, mediante la combinación de diferentes medios, como la "seccional" local del sindicato y las tensiones generadas por la diversidad de intereses. Volviendo a lo planteado por otro entrevistado, surgen diversos aspectos de la acción pública donde se describen formas de construcción de vínculos con la comunidad y los procesos internos de gestión que esto implica. De esta forma, se observa la participación de actores locales de la sociedad civil como las radios y diarios locales, actores del mundo del trabajo como el sindicato y la proximidad con el mundo productivo, entre otros factores. En el plano interno, estas cuestiones pueden verse en el rol del regente con los docentes, asumiendo una determinada perspectiva pedagógica, en términos de reconocimiento de saberes previos y el planteo de situaciones problemáticas. Desde este punto de vista, el proceso de aprendizaje implica la integración de saberes diversos, conceptuales y experienciales.

La capacidad interna de gestión requiere de un soporte técnico-pedagógico que mediante una propuesta técnico-metodológica permita la constitución de los aspectos político-institucionales. Dicha lógica permite cohesionar la ejecución de la política pública con un conjunto de racionalidades “enmarañadas”, en donde la necesidad de la integración de sus actores y sus intereses conlleva la combinación de varios principios de racionalidad. La pertinencia de la expertise en la gestión, según uno de los entrevistados, requiere de un equipo técnico con autonomía, en donde la "operacionalización" de la política como asignación de recursos, permita un sostenimiento de los procesos formativos en el territorio. No obstante, los tiempos formativos a su vez refieren a una necesidad implícita de demandas prácticas para el ámbito de lo laboral.

Entonces, se debe recurrir, además, a una noción de acción pública que no se limite a la intervención de la autoridad. El espacio público (Habermas, 1981) es entendido como un ámbito de confrontación entre interlocutores que tratan de producir acuerdos o compromisos entre ellos y buscar los criterios para precisar la manera en que este compromiso es definido entre diversos actores. Estos criterios se configuran como un referencial global de la política, que se inscribe en una determinada coherencia o "efecto societal". En este marco, los actores, frente a la erosión de las opciones tradicionales de política y ante las nuevas complejidades de la acción pública, tratan de alcanzar coherencia recurriendo a la expertise. Es decir, recurren a un esfuerzo cognitivo (saber experto, técnico-práctico, singular y situado) para obtener los grados de coherencia que requiere su intervención, brindando un direccionamiento de la acción pública, pero sin subordinar las especificidades nacionales y sectoriales a las tendencias "globalizantes" (Verdier, 1997).

Frente a la demanda, por ejemplo, de los avances tecnológicos que conllevan los nuevos modelos, se presentan como un desafío en el cual la formación se muestra como una virtud y a su vez como una necesidad:

"La tecnología avanza tan rápido que no llegamos y me parece que el desafio fuerte en el área automotriz es la capacitación, es la actualización constante. Por eso, ahora compramos un auto híbrido. Tratamos de ir acercándonos a la tecnología, para estar lo 
más cerca posible. Pero me parece que eso es el fuerte, y vos sabes que en el mundo de la mecánica tanto el mecánico se dio cuenta que se tiene que especializar continuamente por eso la formación continua es fundamental. Yo creo que la capacitación es lo que va a hacer la diferencia. (...) Te planta mejor: más sabes, mejor ubicado vas a estar en la vida." (Juan, director CFP SMATA).

Así, el conocimiento situado o expertise permite la conformación de una coherencia en la formación en la cual intervienen intereses múltiples de actores con una red compleja de compromisos. En el caso de la UOCRA, se señalaba en una de las entrevistas, que saber experto y práctica se relacionan, y cuyo espacio toma una centralidad significativa a partir del enfoque pedagógico estructurante. Esto se expresa por ejemplo en una participación significativa en el planteo de situaciones "prácticas" propias del ejercicio profesional, en la carga horaria global, o en el ejemplo de los insumos como elementos claves, estructurantes del acto pedagógico. En los lugares donde estos insumos no están presentes no hay posibilidades para el desarrollo de estas prácticas y se requiere una articulación con proveedores locales para poder desarrollar las prácticas profesionalizantes. Lo mismo con la cuestión del docente/instructor y los procesos de modularización para darle flexibilidad al sistema y actualización a los docentes en cuanto a los desarrollos curriculares. Dicho de otro modo, se requiere una capacidad de gestión importante de un conjunto muy diverso de elementos.

"...gracias a dios hemos armado un buen equipo, esa es nuestra diferencia, que podemos hablar con funcionarios de trabajo, de educación y le hablamos de igual a igual a sus equipos técnicos, pero también necesitas - cuando digo buen equipo también la gestión de compras-, de los elementos de protección personal que vos también tenés que mandar a algún lugar, de los insumos, ver cómo despacho las herramientas (...) Porque esa gente tiene que terminar esa formación porque tiene a la vista la posibilidad de ingresar a una obra. A hívos lo que tenés es que aceitar los elementos que hagan a la práctica. Porque estos cursos son eminentemente prácticos, las dos terceras partes de la carga horaria tienen que estar en las prácticas, necesitas los insumos porque vos no podés guitarrear. Ahi vos tenés que tener los elementos para que la gente vaya y practique y eso implica que la gestión de compra y de aprovisionamiento (...) hay lugares donde vos podés, por cuestiones de espacio físico, no tener todos los insumos porque no los podés tener por cuestiones de seguridad o por cuestiones de espacio, lo que fuera. Entonces tenés que tener el contacto quizás con el corralón que dice bueno, mira, yo te hago este despacho, pero también me lo quedo el tiempo que sea necesario y te la voy entregando a medida en que vos me lo vas requiriendo. Todo eso es gestión, conseguir un docente es gestión, hacer la modularización, es gestión, tratar de que se actualicen los docentes de las actividades que se van incorporando a los desarrollos curriculares que van realizándose es gestión..." (Alejandro, Referente Fundación UOCRA).

Los desafíos que implican los cambios en los procesos de trabajo pueden ser abordados de manera diversa. La perspectiva gremial muestra la idea de reconversión y reaprendizaje de parte de los oficios. El punto de partida está sustancialmente situado en la necesidad de los sujetos. Así, el actor sindical, por el propio modelo de relaciones laborales vigentes en Argentina, ocupa un lugar estratégico en la articulación de recursos, la sostenibilidad de los equipos técnicos y de gestión, para la instrumentación de acciones en el desarrollo de una red de acuerdos y compromisos. En el caso de UOCRA, la asignación de recursos e influjo de proyectos cuenta con la intervención gremial como un elemento estratégico, por su expertise, para dicha articulación e instrumentación de las acciones. De este modo, se relaciona el rol del actor sindical con la asignación de los fondos y de los recursos de acuerdo con la dinámica de los proyectos y las prioridades que se van estableciendo en articulación con los otros actores.

La necesidad de una salida laboral es un factor clave en la actividad y busca cubrir a raíz del perfil de los y las asistentes a sus cursos, sin embargo, desde la organización sindical se plantea otorgar herramientas laborales y extralaborales que formen, pero sin quitar el valor a la experiencia:

“(con referencia a la pregunta en donde se aprende a trabajar) el trabajo lo aprendes trabajando. No tenes vuelta. Nosotros le podemos dar las herramientas, en nuestro centro hacemos las prácticas profesionalizantes donde reparan autos, confias en todo lo que te hacen, se hacen trabajo todo, pero el mundo del trabajo es otro. Tratamos de enseñárselo: la puntualidad, la forma correcta de vestirse, el trato, las buenas prácticas, la seguridad. Pero el trabajo diario, las dificultades diarias y todo lo demás no lo podés aprender en otro lado que no sea trabajando. No hay forma. Podes estar bien capacitado y te va a resultar mucho más cómodo y práctico, y vas a aprender mucho más rápido cuantos más conocimientos traigas y si en el centro, por ejemplo, que desarman autos y todo lo demás, cuando llegue el momento de tornear no le va a costar nada porque ya lo estuvo haciendo y el auto en el centro llega con una falla y se tiene que ir funcionando tal cual como si fuese en un taller o en una concesionaria. Pero el contacto con los otros compañeros porque no es lo mismo ser un compañero de estudio que ser un compañero de trabajo. El trato con los 
empleadores que es muy diferente donde ya van a tener deberes y derechos. Nosotros le explicamos toda la parte sindical también, pero también saben que tienen deberes y derechos. Pero eso se aprende en el trabajo, no hay forma. Yo creo que no hay ninguna. El tipo que aprendió toda su vida trabajando, de ahi sacamos las competencias laborales donde certificamos al trabajador en su puesto de trabajo. Encontramos esa manera que nos parece bastante interesante donde el tipo recibe un certificado por los saberes que el adquirió en su experiencia laboral" (Juan, director CFP SMATA).

De las palabras del entrevistado puede inferirse que la adquisición de conocimiento de las relaciones que se dan en el mundo del trabajo conforma contenidos extracurriculares cuyo contenido se encuentra impregnado de las nociones propias del gremio. La relación en los espacios formativos, en cuanto a los contenidos que se ponen en juego, deja entrever la idea de necesidad de adquisición de ciertos capitales y credenciales que exceden los conocimientos técnicos. Dirigido a un específico campo ocupacional, desde este dispositivo institucional -la red de UOCRA y SMATA- se plantean propuestas formativas relacionadas con los cambios en el sector y con la reconversión tecnológica y de las calificaciones de los trabajadores.

En este marco, hay que considerar que la formación tiene lugar en contextos de baja previsibilidad, inestables y atravesados por esquemas poco rutinarios e inciertos para el ejercicio de las capacidades profesionales. La noción de articulación es nodal para reconstruir puntos de conexión que garanticen el cumplimiento de los acuerdos. Esto supone cierta tensión entre los contextos formativos y las formas sistemáticas de seguimiento del cumplimiento de los compromisos asumidos por los actores (Verdier, 1997).

La construcción coherente entre el saber técnico experto y los procesos de aprendizaje requiere la tarea conjunta de los actores, lo que implica una tarea de definiciones y redefiniciones, en donde las capacidades y saberes de gestión revalorizan estos procesos de mejora, aprendizaje y ajustes en la política (Verdier, 1997). En este sentido, uno de los entrevistados de UOCRA señalaba respecto de la articulación entre la educación de adultos y la Formación Profesional cómo cuenta la complejidad de los dispositivos institucionales que requieren las políticas de FP debido a la diversidad de demandas, provocando itinerarios muy dispares entre los asistentes a los cursos. El entrevistado marcaba que la dificultad de pérdida de matrícula de la formación de adultos está dada dentro de un sector de actividad específico con el desarrollo de saberes de oficio. Esto se convertía en una controversia por parte de los asistentes de la utilidad de la oferta formativa brindada por la institución. A diferencia de la propuesta del CFP que se correspondía de modo mucho más "claro" y "firme" con cierta dinámica de reconversión tecnológica y de las calificaciones en la industria de la construcción. Por su parte, los docentes de FP sostenían que, dada esta ausencia de competencias básicas, debían dedicar la primera etapa de los cursos a "reforzar" elementos matemáticos de base. A partir de este conjunto de demandas es que desarrollaron la iniciativa piloto de "articulación cruzada". Esto implicó un conjunto de acciones en materia curricular, combinando formación en oficios y "terminalidad” educativa. De esta forma, se construyó una propuesta formativa "innovadora" basada en la búsqueda de respuestas alternativas frente a la heterogeneidad de situaciones sociolaborales que se presentan en cada institución.

La gestión y ejecución de las políticas de FP por parte del actor sindical, supone la generación y acumulación de saberes y capacidades técnicas, así como el desarrollo de lobby para poder incidir en el direccionamiento de las políticas públicas. Esto muestra, de acuerdo con lo señalado por los entrevistados, una alta flexibilidad y una oferta diferencial de la política, respecto de beneficiarios con trayectorias diversas, mostrando la "efectividad", pero a la vez las limitaciones, de los esquemas tripartitos para la ejecución de la política. Así la gestión del saber y su red, como espacio de construcción de saber colectivo producto de la interacción, se configura como una zona ampliada de acción pública de formación.

\section{Comentarios FinALES}

En este trabajo se analizó el rol del actor sindical y la práctica de gestión de dispositivos de formación a nivel "macro", con eje en su dimensión política a partir del concepto de acción pública de Verdier, destacando su centralidad en la práctica. Esta práctica es vista en la interacción del sindicato con el Estado y 
el campo de las políticas públicas y con los actores del empresariado. Se analizó también las modalidades de construcción de estos vínculos compatibilizando las demandas del sector productivo con la mejora en las condiciones de inserción sociolaboral, analizando el papel del sindicato y sus formas de interpelar al actor estatal en cuanto a las políticas de FP. Considerando la acción pública de FP como una red sectorial de compromisos, los entrevistados brindan ejemplos enfocando la formación con el campo profesional y con la construcción de un dispositivo institucional que brinde los recursos necesarios para la ejecución de la política.

Al abordar la red de CFP de UOCRA y SMATA y cómo ésta fue diversificando sus servicios de formación y su oferta formativa se señalaron tensiones presentes en la articulación entre los campos de la educación y el trabajo para desarrollar un proceso "eficiente" en la gestión del saber. Se asocia a esto un concepto ampliado de racionalidad y la inclusión de la instancia política e institucional en el desarrollo de estos sistemas de desarrollo, innovación e integración social. En este sentido, se "dialoga" con el problema del desarrollo desde un enfoque que se acerca, desde una mirada sectorial y acotada, a aquellas perspectivas que consideran al saber cómo el insumo fundamental de la economía global, donde el factor estratégico para la competitividad es el aprendizaje en tanto instancia colectiva de construcción de saber. Por otra parte, se considera que la construcción de saber responde a un tipo de racionalidad ampliada y comunicativa que implica interacción entre actores diversos del mundo laboral y productivo, asociado al desarrollo de un conjunto de valores profesionales que orientan la actividad, por lo que resultaron centrales en el estudio la dimensión cultural y político-institucional.

Según los entrevistados, esto requirió desarrollar una actualización de procedimientos y estructuras institucionales y el desarrollo de capacidades especializadas para poder incidir sobre estas políticas públicas, en la búsqueda por mejorar las posibilidades de inserción laboral y productiva de los trabajadores del sector que asisten a los cursos. A partir de ello se constituyó la red como sistema, un ámbito de confluencia del sistema educativo, de las políticas de empleo, de las políticas de desarrollo de tecnología, de las relaciones laborales y del fomento de las actividades productivas. Esta cercanía con el mundo laboral y productivo, potencia los aprendizajes por la experiencia, al considerar en un mismo espacio formativo -o zona de desarrollo, innovación y aprendizaje-, las dimensiones productivas, laborales, tecnológicas y sociales.

Es en este esquema sectorial, donde los actores del mundo del trabajo representativos de esta actividad, el sindicato principalmente, brindan un marco de inserción social, territorial y sectorial a las instituciones de formación, facilitando la gestión y resolución de los conflictos, disfunciones y desajustes dentro del marco más general de las relaciones laborales y del mundo del trabajo. Esto se traduce en un esquema complejo de FP, a partir de procesos de mejora y aprendizaje permanente, lo que supone para el sindicato, muchas veces, modificar la propia acción en la interacción con los otros actores (Estado, cámaras empresarias) y en el propio proceso de gestión institucional.

De este modo, en la perspectiva de los referentes sindicales, se va configurando una mirada donde las contrapartes del capital y el trabajo resultan esenciales para la ejecución de las políticas, lo que permite construir compromisos sólidos basados en intercambios permanentes que dan legitimidad y credibilidad a estos sistemas sectoriales y nacionales. De esta forma, según los entrevistados, se logran moderar las tensiones entre los actores, en un marco de negociación dentro del campo de las políticas públicas de educación y trabajo.

\section{ReFERENCIAS}

Baraldo, N. (2020). Movimiento obrero y educación. Sentidos y estrategias en torno a los Centros Educativos de Nivel Secundario. Argentina 1970-1974. Trabajo y Sociedad, 21, (34), 179-198

Bourdieu, P. y Passeron, J.C. (1977). La reproducción: elementos para una teoría del sistema de enseñanza. Barcelona: Laia.

Briasco, I. (2014). Argentina. Sistema nacional de cualificaciones. Informe nacional eurosocial. 
Corica, A. M. y Otero, A. E. (2020). Cambios en las transiciones educación-trabajo. Egresados del secundario del Gran Buenos Aires. Revista de Ciencias Sociales, 33 (47), 139-161.

Dussel, I. (2020). La escuela en la pandemia. Reflexiones sobre lo escolar en tiempos dislocados, Ponta Grossa, Práxis Educativa, 15, 2016482, 1-16. https://doi.org/10.5212/PraxEduc.v.15.16482.090

Ferrer, A. (2004). La economía argentina. Buenos Aires: Fondo de Cultura Económica

Filmus, D (2014). La universalización del secundario en América Latina: una meta necesaria para la inclusión social y la ampliación de derechos de los jóvenes. Buenos Aires: Mimeo

Gallart, M. (2002). Veinte años de educación y trabajo. Montevideo: CINTERFOR-OIT

Giampaoletti, N. (2020). La Escuela Técnica Industrial y las disputas por la legitimación de los saberes del trabajo. Revista de la Escuela de Ciencias de la Educación, 1 (15), 44- 5

Habermas, J. (1981). Teoría de la Acción Comunicativa. Madrid: Editorial Trotta

Jacinto, C. y Chitarroni, H. (agosto, 2009). Precariedades, rotación y acumulación en las trayectorias laborales juveniles. Trabajo presentado en 9 Congreso Nacional de Estudios del Trabajo, ASET, Buenos Aires

Jacinto, C. (2010). La construcción social de las trayectorias laborales de jóvenes: políticas, instituciones, dispositivos y subjetividades. Buenos Aires: Teseo- Ides

Jacinto, C. (2016). Protección social y formación para el trabajo de jóvenes en la Argentina reciente: entramados, alcances y tensiones. Buenos Aires: Instituto de Desarrollo Económico y Social

Lundvall, B. (2009). Sistemas Nacionales de Innovación. Buenos Aires: UNSAM EDITA

Millenaar, V. (2017). Políticas de empleo con enfoque de género: formación laboral en oficios no tradicionales para mujeres. Cadernos pagu, (51). https://doi.org/10.1590/18094449201700510014

Miranda, A. (2016). Transiciones juveniles, generaciones sociales y procesos de inclusión social en Argentina postneoliberal. Linhas Criticas, 22 (47), 130-149

Miranda, A. y Corica, A. (2018). Gramáticas de la Juventud: reflexiones conceptuales a partir de estudios longitudinales en Argentina. En Corica A., A. Freytes Frey y A. Miranda (compiladoras). Entre la educación y el trabajo: la construcción cotidiana de las desigualdades juveniles en América Latina. Buenos Aires: CLACSO

Neffa, J. (2000). Las innovaciones cientificas y tecnológicas: Una introducción a su economía política. Buenos Aires: Lumen-Humanitas,

Puiggrós, A. y Carli, S. (1995). Discursos pedagógicos e imaginario social en el peronismo, 1945-1955. Buenos Aires: Galerna

Riquelme, G. (2006). La relación entre educación y trabajo: continuidad, rupturas y desafíos. Anales de la educación comú, 2 (5), 68-75

Riquelme, G. C., Herger, N. y Kodric, A. I. (2017). Deuda social con la educación y formación de jóvenes y adultos: accesibilidad, barreras y escenarios complejos. Revista Brasileira de Politica e Administração da EducaçãoPeriódico cientifico editado pela ANPAE, 33 (1), 37-54

Tedesco, J. C. (1986). Educación y sociedad en la Argentina:(1880-1945). Buenos Aires: Ediciones Solar

Tenti Fanfani, E. (2007). La escuela y la cuestión social: ensayos de Sociología de la educación. Buenos Aires: Siglo veintiuno editores

Vasilachis, I. (1992). Métodos cualitativos I. Los problemas teórico-epistemológicos. Buenos Aires: Centro Editor de América Latina

Verdier, E. (diciembre, 1997). Recomposición de la acción púbica para el empleo y la formación profesional. Trabajo presentado en seminario organizado por el Conicet y el PIETTE, Buenos Aires

Wainstok, C. (julio, 2013). Descolonizaciones. Trabajo presentado X Jornadas de Sociología. Facultad de Ciencias Sociales, Universidad de Buenos Aires

Yoguel G. (2000). Algunas reflexiones acerca de la importancia de losprocesos de aprendizaje en el desarrollo de las ventajas competitivas de los agentes. Buenos Aires: Littec 


\section{Notas}

1 Se agradecen las observaciones y comentarios realizados en los dictámenes, así como los comentarios realizados por el equipo editorial. Dichos aportes han contribuido notablemente a la mejora del artículo. No obstante, las opiniones expresadas son de exclusiva responsabilidad de los autores. 